\title{
Amadeus
} International Multidisciplinary Journal IISS 2525-8281

DOI: $10.14295 /$ aimj.v4i7.69

\section{Use of Trilingual Posters as Access Technology to Prenatal of Immigrants}

Rafael Zaneripe de Souza Nunes ${ }^{1}$, Stephane Catharine Zavadil ${ }^{2}$ Jacks Soratto ${ }^{3}$, Karin Martins Gomes ${ }^{4}$, Graziela Amboni ${ }^{5}$, Caroline Zaneripe de Souza ${ }^{6}$

\begin{abstract}
Immigration around the world is an important discussion to study in the context of Public Health. To Brazil, one of the biggest challenges is verbal communication to Haitian and Ghanaian immigrants, as most of them don't speak the language of the immigrant region. Realizing the difficulty in communication and guidance within Public Health, a trilingual information poster was created to serve as a technology for access to these users and to facilitate the work of health professionals in prenatal care. The poster was produced through the performance of the Multiprofessional Residency in Public Health, of the Postgraduate Program in Public Health (PPGSCOL) present at the University of Santa Catarina (UNESC).
\end{abstract}

Keywords: Public Health, Immigrants, Equity in Access to Health Services, Prenatal care.

\section{Uso de Cartazes Trilingue como Tecnologia de Acesso ao Pré-Natal de Imigrantes}

Resumo: A imigração em todo o mundo é uma discussão importante a ser vista com um olhar mais atento no contexto de Saúde Pública. Para o Brasil, um dos maiores desafios é a comunicação verbal para os imigrantes haitianos e ganeses, visto que estes, em sua maioria, não falam o idioma da região imigrada. Percebendo a dificuldade na comunicação e orientação dentro da Saúde Coletiva foi criado um cartaz informativo trilíngue para servir como tecnologia de acesso a esses usuários e facilitar o trabalho dos profissionais de saúde na realização do pré-natal. A produção do cartaz se deu por meio da atuação da Residência Multiprofissional em Saúde Coletiva, do Programa de Pós-Graduação em Saúde Coletiva (PPGSCOL) presente na Universidade do Extremo Sul Catarinense (UNESC).

Palavras-chave: Saúde Pública, Imigrantes, Equidade no Acesso aos Serviços de Saúde, Cuidado Pré-Natal.

\footnotetext{
${ }^{1}$ Resident Psychologist in the University of Southern Catarinense. rafaelzaneripe.psico@gmail.com;

${ }^{2}$ Resident Psychologist in the University of Southern Catarinense. stephaneecz@ hotmail.com;

${ }^{3}$ Professor in the University of Southern Catarinense. jacks@unesc.net;

${ }^{4}$ Professor in the University of Southern Catarinense. karin@unesc.net;

${ }^{5}$ Professor in the University of Southern Catarinense. gam@unesc.net;

${ }^{6}$ Psychologist by the Federal University of Santa Catarina. carolzpsi@gmail.com.
} 


\section{Introdução}

Os fluxos imigratórios ao redor do mundo são um dos assuntos mais discutidos e noticiados na atualidade. Além de ser um tema muito discutido e polêmico, também levanta questionamentos por parte da população e gestão pública de como se lidar com essa realidade. Para Silva e Lima (2017), a imigração sempre existiu e foi motivo de preocupação. Mas atualmente esses fluxos imigratórios estão tomando proporções muito maiores do que foram em períodos anteriores. Da mesma forma que brasileiros vão para os Estados Unidos e Europa buscar uma vida melhor, africanos e latino americanos buscam vir ao Brasil com a esperança de ter uma vida digna para si e sua família.

As causas por trás do fenômeno migratório são as mais diversas possíveis, e podem estar ligadas a fatores econômicos, sociais, políticos, filantrópicos, culturais, religiosos e outros (LIMA e SILVA, 2017; DUTRA e GAYER, 2015). Os estrangeiros podem ser definidos em dois tipos, os residentes e os não residentes do país (em processo de trânsito). Mas, independentemente do tipo, todo estrangeiro deve possuir uma condição jurídica que respeite sua dignidade (LIMA e SILVA, 2017). Dentre as principais dificuldades enfrentadas pelos imigrantes ao chegarem a um país, o idioma é uma das maiores. Com relação a isso, Dutra e Gayer (2015, p.7-8) afirmam:

\footnotetext{
"Um dos maiores desafios para os imigrantes é o aprendizado da língua portuguesa, (A dificuldade de comunicação por não poder estudar a língua portuguesa ou a sua dificuldade de comunicação os isola, tem efeitos no desempenho do trabalho, na busca de serviços, assim como para sua alimentação e ir nos postos de saúde),a falta de sensibilização dos poderes públicos municipais e estadual que não oferecem espaços físicos e docentes, a inexistência de um método eficiente a ser utilizado pelos monitores em todos os estados, e o desnível cultural entre os migrantes"
}

Para os imigrantes haitianos e ganeses essa realidade não é diferente, Dutra e Gayer (2015) salientam que uma das maiores barreiras que esses enfrentam também é com relação à língua, pois um bom número desses imigrantes falam crioulo haitiano, francês e inglês. Por cota disso, quando chegam ao Brasil, tendem a andar juntos, formando guetos. Ocorre também um choque cultural, pois países como gana e Haiti são pequenos, e os imigrantes acabam se assustando com a dimensão do Brasil, e todos esses fatores combinados tornam o processo de adaptação lento e demorado. 


\section{Caracteristicas Linguísticas e Sociais do Processo Imigratório de Haitianos e Ganeses}

O povo haitiano é diglóssico, ou seja, em seu pais há o registro linguístico do criolo haitiano e o francês coexistindo, sendo seu uso dependente da situação comunicativa, entretanto a maioria da população fala o criolo haitiano, mas compreende o francês. Já o povo ganês é composto por um enorme número de tribos, cada uma com sua cultura e idioma diferentes. A língua oficial de Gana é o inglês, mas a maioria da população fala o akan. Sendo assim, o povo ganês em sua maioria é bilíngue, utilizando o seu idioma para falar dentro do seu grupo étnico/cultural, e akan/inglês para se comunicar com pessoas de fora (Dutra e Gayer, 2015).

É sabido que muitos brasileiros sofrem com o desemprego, e os imigrantes ao virem para o Brasil sofrem também, pois suas esperanças acabam sendo frustradas e acabam encontrando situações demasiadamente precárias, mas essas situações ainda são melhores do que as de seus países de origem. Apesar disso, o ser humano não pode viver com menos do mínimo necessário para ter uma vida digna, por isso o Estado deve criar políticas públicas eficazes para proteger aqueles que estejam em situação de vulnerabilidade. (LIMA e SILVA, 2017)

Dessa forma, precisamos discutir acerca de igualdade e as formas de lidar com situações de vulnerabilidade. Para que isso seja efetivado de maneira real, Dutra e Gayer (2015) salientam que se deve dar tratamento "igual aos iguais e desigual aos desiguais", ou seja, as pessoas por serem diferentes têm necessidades diversas e a lei demanda que as condições apropriadas de atendimento sejam cumpridas de acordo com as particularidades de cada individuo. A inclusão pode ser configurada no principio da igualdade, pois a diversidade requer peculiaridade de tratamento.

De qualquer maneira, a imigração é motivo de preocupação, pois o Brasil está inserido numa nova seara das migrações internacionais, e isso exige um maior debate nos meios acadêmicos e na esfera da gestão pública sobre o que pode ser feito para inserir esses imigrantes, evitando e combatendo o trabalho escravo, preconceito e xenofobia (UEBEL, 2016; DUTRA e GAYER, 2017). Um dos fatores mais atrativos para os imigrantes haitianos na escolha do Brasil como destino é a lógica econômica. Juntamente a isso, vemos as facilidades concedidas pelo governo brasileiro para a entrada e 
instalação de haitianos (NIETO, 2014), talvez esses fatores se relacionem também ao caso da imigração ganesa.

Para Pedemonte, Amode e Rencoret (2015), imigrantes haitianos, por exemplo, assumem altos custos para se estabelecer em um país (como o racismo e a precariedade de trabalho) e se possível ganharem o status de sujeito de valor e bons trabalhadores. Demonstram em meio a todas essas coisas uma grande capacidade adaptativa e, sobretudo resiliência.

\section{Imigrantes no Sistema Ùnico de Saúde}

Podemos ver que é inegável a complexidade da onda imigratória e seus diversos desdobramentos. Uma das consequências causadas por esse fenômeno acaba se refletindo no SUS (Sistema Único de Saúde), pois sendo um Sistema Público de Saúde suas ações abarcam a todos que estão em seu território, inclusive aos imigrantes presentes no Brasil. Na prática profissional em Unidades Básicas de Saúde e outros pontos de atenção do SUS, criam-se tensões decorrentes das dificuldades de comunicação dos profissionais com os imigrantes. Entretanto, muitos podem se perguntar: o que o SUS tem a ver com isso? Ou até mesmo: o SUS tem a obrigação de atender a esses imigrantes? Para respondermos a essas perguntas, é necessário contextualizarmos o que é o SUS e como foi seu surgimento.

No ano de 1988, a partir da instauração da nova Constituição Federal, em um contexto que se buscava a implantação de um estado de bem-estar social tendo a saúde como um direito de cidadania, dava-se origem ao processo de criação de um sistema público, universal e descentralizado de saúde (Paiva e Teixeira, 2014). Após décadas de luta pelo Movimento da Reforma Sanitária, o Sistema Público de Saúde foi criado. O SUS teve sua concepção com base na Constituição Federal de 1988 e sua consolidação pelas Leis 8.080 e 8.142. Numa análise de suas características a partir de sua colocação constitucional, esse sistema de saúde tem como essência a ideia de que Saúde é Direito do Cidadão e Dever do Estado (CARVALHO, 2013).

A Constituição Federal de 1988 , em seu $197^{\circ}$ artigo, afirma que as ações e serviços públicos de saúde devem integrar uma rede regionalizada e hierarquizada, ou seja, existem níveis de complexidade de atenção na forma como o Sistema Público de Saúde funciona. O Decreto 7508/11 vai de encontro com o que foi exposto sobre os aspectos de hierarquia e regionalidade da rede que compõe o Sistema Único de Saúde, 
que deve ser organizado em regiões de saúde que contemplem no mínimo ações de atenção primária, urgência e emergência, atenção psicossocial, atenção ambulatorial especializada e hospitalar, e vigilância em saúde. (BRASIL, 1988, 2011)

Nos sistemas fragmentados de atenção a saúde, os pontos de atenção são isolados e não se comunicam entre si, o que leva a uma incapacidade por parte desse sistema de prestar uma atenção continua e integral a população. Nesses sistemas, a atenção primária não tem uma comunicação dinâmica com a atenção secundária, e ambos não se comunicam com a atenção terciaria ou com os sistemas de apoio (MENDES, 2010). De acordo com Arruda (2015), podemos perceber que as Redes de Atenção (RAS) funcionam ao contrário de um sistema fragmentado de atenção a saúde, pois se configuram a partir de múltiplas conexões e inter-relações, funcionando numa ótica multidimensional, pois conecta pontos que possuem características distintas, envolvendo diferentes tipos de profissionais e usuários.

O Sistema Único de Saúde (SUS) desde sua criação se pautou no bem-estar social e na saúde como um direito, sendo o Estado responsável pela mobilização dos recursos e efetivação desses princípios. Além disso, o SUS compreende uma rede complexa e interligada de pontos de atenção, sendo assim, o individuo ao ser atendido não se torna responsabilidade de um ponto de atenção, mas da rede como um todo. Podemos perceber através de Santos (2016), que apesar de ter se passado mais de duas décadas desde a criação do SUS, os desafios para efetivação dos princípios de universalidade, integralidade e equidade ainda persistem.

Se nosso Sistema Público de Saúde é universal, logo suas ações se estendem a todos, inclusive aos imigrantes, seja ele haitiano, ganes ou qualquer outro. Se o Estado está responsável pelo cuidado da saúde dessas pessoas, é imprescindível que se mobilize os recursos necessários e criem-se tecnologias de acesso para que a rede de atenção e os profissionais se adaptem a essa nova realidade. Segundo Santos (2016), para que a incorporação dos princípios do SUS se efetive é imprescindível reconhecer que há certos grupos sociais que enfrentarão mais dificuldades no acesso aos serviços de saúde da rede. Esse é o ponto inicial para se pensar em alternativas que transponham as barreiras de acesso desses imigrantes ao sistema de saúde.

Para Venters e Gany (2009), um problema comum envolvendo imigrantes africanos que não possuem documentação, é que muitos evitam procurar cuidado médico em decorrência dessa situação. Deve-se levar em consideração também o crescente e recente sentimento anti-imigratório como um aspecto importante, que juntamente com 
outros fatores ganha e perde significância ao longo do tempo, mas ajuda a compreender questões relativas ao acesso a saúde dessas populações (REYNOLDS E CHILDERS, 2019).

O Brasil se tornou um destino atrativo para migração, tanto para africanos, quanto para latino-americanos e asiáticos. O sistema público de saúde deve preparar-se de maneira adequada pare atender aos problemas de saúde que emergirem dessa onda imigratória (RAWLINSON et.al, 2014).

\section{Imigrantes e Cuidado Pré-Natal nas Unidades Básicas de Saúde}

Dentro das unidades básicas de saúde em regiões que possuem imigrantes a dificuldade na comunicação se instaura devido às diferenças linguísticas. No município de Criciúma os profissionais de saúde se deparam com essa demanda proveniente de imigrantes haitianos e ganeses. Há bairros com uma grande concentração desses imigrantes, entretanto não se tem uma estimativa percentual acerca do número exato destes.

De acordo com Newbold e Danforth (2003), o estado de saúde está associado à qualidade de vida e ao uso de serviços de saúde formais e informais. No geral, os imigrantes parecem ser mais saudáveis do que a população nascida local, em virtude de sua raça. No entanto, de acordo com os autores, ao longo do tempo, a saúde é afetada devido aos efeitos da imigração.

Ainda, Gushulank e MacPherson (2006) ressaltam que a saúde dos imigrantes é resultado de vários fatores, como: ambientais, econômicos, genéticos e socioculturais relacionado a quando as pessoas migraram, onde e como eles viviam em seu país natal original, e como e por que eles migraram. A sua saúde é também influenciada por questões pós-migratórios que envolvem a integração no novo local de residência, emprego, educação e pobreza, bem como acessibilidade e capacidade de resposta dos profissionais de saúde e resposta do sistema de saúde às necessidades de saúde dos imigrantes.

Outra demanda surge quando esses imigrantes começam a se estabelecer nas cidades: a família começa a se expandir. Vendo a possibilidade de uma vida melhor e mais digna do que em seus países os imigrantes começam a constituir famílias. Nessa perspectiva, Batista e Gugelmin (2018) apontam que após o processo de reunificação 
familiar dos imigrantes nos pais, há uma elevação do número de nascimento de crianças oriundas de mães haitianas.

As imigrantes (agora gestantes) comparecem as Unidades Básicas de Saúde em busca de suporte. Naturalmente as enfermeiras se deparando com uma gestante em sua frente realizam o acolhimento e começam a executar e/ou agendar a consulta de pré-natal, buscando dar as devidas orientações. Mas a grande questão é que os profissionais não contavam com as dificuldades de comunicação oriundas da diferença linguística.

Apesar do constante aumento do número de nascimentos de crianças de imigrantes haitianas no Brasil, não há estudos que avaliem questões concernentes ao cuidado pré-natal a essa população (BATISTA \& GUGELMIN, 2018), não encontramos também estudos relativos às gestantes ganesas.

\section{Cartazes como Tecnologia de Acesso para Gestantes Imigrantes}

Percebendo as dificuldades que alguns profissionais poderiam sofrer ao orientar essas usuárias sobre questões de saúde e direito da gestante, criamos um cartaz informativo trilíngue baseado nessa temática para servir como tecnologia de acesso a esses usuários e facilitar o trabalho dos profissionais de saúde na realização do pré-natal. Com relação às dificuldades de atender a esse público Topa, Neves e Nogueira (2013, p.336) salientam que os/as "profissionais de saúde não estão devidamente preparados/as para atuar junto de populações imigrantes, contribuindo muitas vezes para o reforço de constrangimentos sentidos".

Um dos principais obstáculos das politicas públicas voltadas à saúde sexual e reprodutiva dos imigrantes é a falta de coordenação dos governos locais frente à problemática. As populações migrantes e de grande mobilidade geográfica geralmente apresentam dificuldade de acesso aos serviços de saúde (MARTINS-PIZARRO \& REBOIRAS-FINARDI, 2010). Para Topa, Neves e Nogueira (2013), as mulheres migrantes enfrentam vários desafios quanto à acessibilidade aos cuidados de saúde. Tais dificuldades intensificam-se principalmente nos cuidados a saúde sexual e reprodutiva, sendo a gravidez e a maternidade os períodos com maior vulnerabilidade aos agravos à saúde, tanto para a gestante quanto para seus filhos.

Sendo assim, pensando na lógica da acessibilidade aos cuidados de saúde sexual e reprodutiva, a produção desse cartaz se deu por meio do Programa de Pós-Graduação em Saúde Coletiva (PPGSCOL) presente na Universidade do Extremo Sul Catarinense 
(UNESC) que contava com a atuação da Residência Multiprofissional em Saúde Coletiva. Os profissionais presentes no programa tiveram um olhar crítico frente a essa demanda no município, e por meio disso, em parceria com Prefeitura Municipal de Criciúma se trabalhou na construção e distribuição dos cartazes para auxiliar na problemática presente.

Figura 1 - Cartaz para gestantes imigrantes.

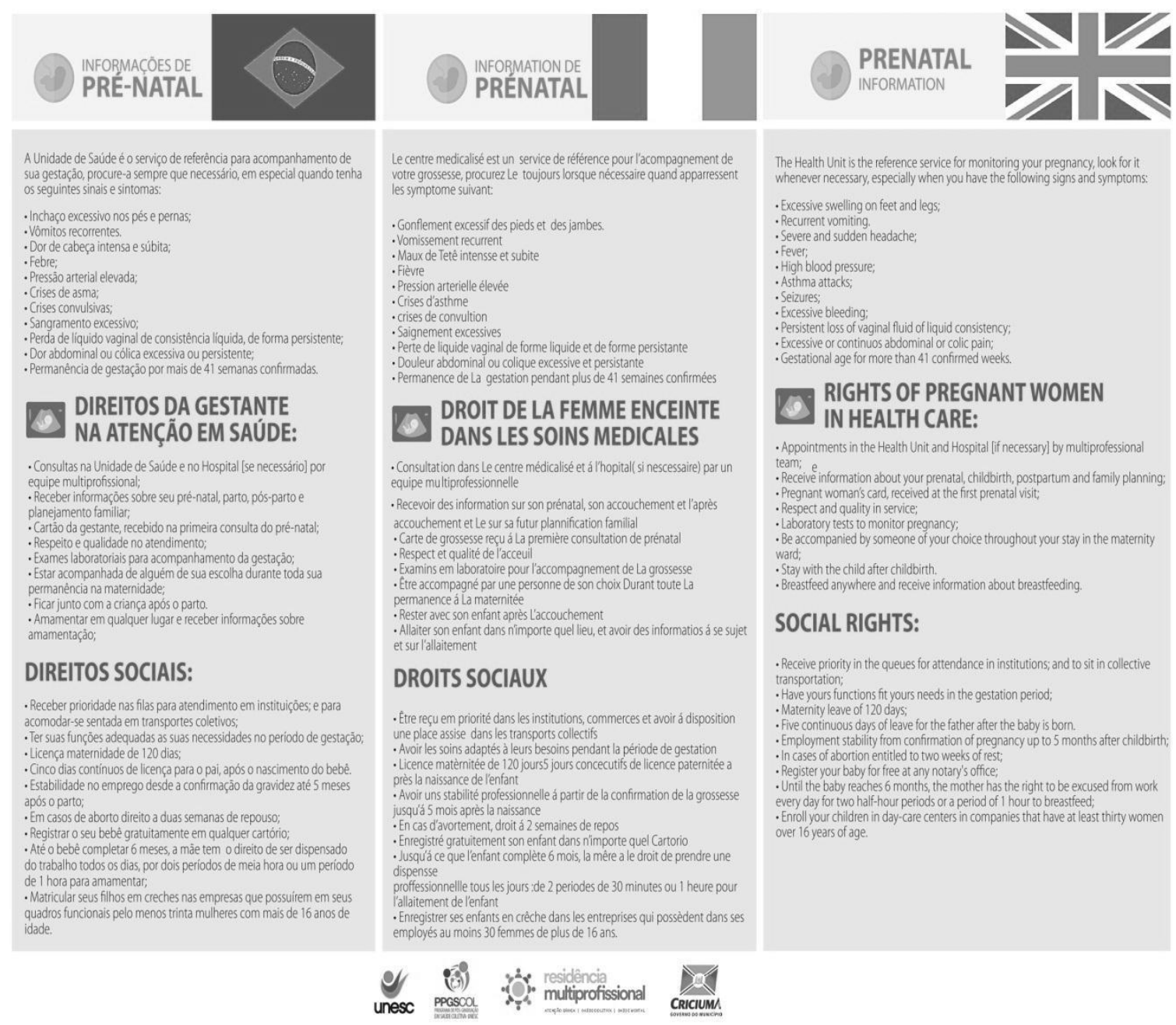

Fonte: Próprios Autores do Estudo.

A usuária e seu parceiro podem apontar para um dos itens em um determinado tópico do cartaz para informar algo que esteja acontecendo com a gestante. Por meio disso, o profissional de saúde pode olhar o item paralelo equivalente na língua portuguesa e identificar o sintoma ou situação pela qual a usuária está passando/sentindo. Isso está de acordo com a sugestão feita por Santos (2016), em que ações simples podem impactar e 
facilitar o acesso dos imigrantes aos serviços de saúde através da sensibilização das diferenças culturais entre profissionais, gestores e usuários do sistema.

\section{Considerações Finais}

A imigração é um tema que deve ser discutido e visto com um olhar mais atento dentro da atuação dos profissionais na Saúde Coletiva, visto que uma das maiores dificuldades de ganeses e haitianos é a comunicação verbal. Os cuidados a saúde sexual e reprodutiva acabam sendo afetados também por consequência da dificuldade em entender o idioma local. Nessa perspectiva, a gravidez e a maternidade acabam sendo os períodos com maior vulnerabilidade aos agravos à saúde, tanto para a gestante quanto para seus filhos.

Nesse sentido, o cartaz trilíngue serviu como informativo e tecnologia de acesso a esses usuários imigrantes, além de facilitar o trabalho dos profissionais de saúde na realização do pré-natal. Além disso, a produção deste cartaz pode servir como porta de entrada para a reflexão de outras estratégias para o cuidado de imigrantes haitianos e ganeses na Atenção Básica a Saúde e nos outros pontos de saúde do sistema público.

\section{Referências}

Arruda, C. et al. (2015). Redes de atenção à saúde sob a luz da teoria da complexidade. Esc. Anna Nery, Rio de Janeiro, v. 19, n. 1, p. 169-173.

Batista, D. R. R.; Guglemin, S. A.; Muraro, A. P. (2018). Acompanhamento pré-natal de mulheres brasileiras e haitianas em Mato Grosso. Rev. Bras. Saude Mater. Infant., Recife, v. 18 , n. 2 , p. 317-326.

Brasil. (1988). Constituição da República Federativa do Brasil. Brasília: Senado Federal.

Brasil. (2011). Decreto $n^{\circ}$ 7.508, de 28 de junho de 2011. Regulamenta a Lei no 8.080, de 19 de setembro de 1990, para dispor sobre a organização do Sistema Único de Saúde SUS, o planejamento da saúde, a assistência à saúde e a articulação interfederativa, e dá outras providências.

Carvalho, G. (2013). A saúde pública no Brasil. Estud. av., São Paulo, v. 27, n. 78, p. 7-26.

Dutra, C. F.; Gayer, S. M. (2015). A inclusão social dos imigrantes haitianos, senegaleses e ganeses no Brasil.

Gushulak, B.D.; MacPherson, D.W. (2006). Migration medicine and health: principles and practice. Hamilton $(\mathrm{ON})$ : BC Decker. 
Lima, S. S. de; Silva, L. M. M. da. (2017). Os imigrantes no Brasil, sua vulnerabilidade e o princípio de igualdade. Revista Brasileira de Politicas Públicas. Brasília: Vol. 7, Ed. 2: 385.

Martinez-Pizarro, J.; Reboiras-Finardi, L. (2010). Migración, derechos humanos y salud sexual y reproductiva: delicada ecuación en las fronteras. Pap. poblac, Toluca , v. 16, n. 64, p. 09-29, jun.

Mendes, E. V. (2010). As redes de atenção à saúde. Ciênc. saúde coletiva, Rio de Janeiro, v. 15, n. 5, p. 2297-2305, Aug.

Newbold, KB; Danforth J. (2003). Health status and Canada's immigrant population. Soc. Sci Med, 57:1981-95.

Nieto, C. (2014). Migración haitiana a Brasil: Redes migratorias y espacio social transnacional. CLACSO, Buenos Aires.

Paiva, C. H. A.; Teixeira, L. A. (2014) Reforma sanitária e a criação do Sistema Único de Saúde: notas sobre contextos e autores. Hist. cienc. saude-Manguinhos, Rio de Janeiro, v. 21, n. 1, p. 15-36, Mar.

Pedemonte, N. R.; Amode, N. R., Jorge V. (2016). Racismo y matrices de "inclusión" de la migración haitiana en Chile: elementos conceptuales y contextuales para la discusión, Polis, $v$. 42.

Rawlinson, T.; Siqueira A.M.; Fontes, G., Beltrão, R.P.L.; Monteiro, W.M.; Martins, M., et al. (2014) From Haiti to the Amazon: Public Health Issues Related to the Recent Immigration of Haitians to Brazil. PLoS Negl Trop Dis 8(5): e2685.

Reynolds, M. M.; Childers, T. B. (2019). Preventive Health Screening Disparities Among Immigrants: Exploring Barriers to Care. Journal of Immigrant and Minority Health.

Santos, F.V. dos. (2016). A inclusão dos migrantes internacionais nas políticas do sistema de saúde brasileiro: o caso dos haitianos no Amazonas. Hist. cienc. saude-Manguinhos, Rio de Janeiro, v. 23, n. 2, p. 477-494, June.

Topa, J.; Neves, S.; Nogueira, C. (2013). Imigração e saúde: a (in)acessibilidade das mulheres imigrantes aos cuidados de saúde. Saude soc., São Paulo, v. 22, n. 2, p. 328-341, June.

Uebel, R. R. G. (2016). Panorama e perfil da imigração senegalesa no Rio Grande do Sul no início do Século XXI. Boletim Geográfico do Rio Grande do Sul, Porto Alegre, n. 28, p. 56-77, set.

Venters, H.; Gany, F. (2009). African Immigrant Health. Journal of Immigrant and Minority Health, 13(2), 333-344.

\section{How to cite this article (APA format):}

Nunes, Rafael Zaneripe de Souza; Zavadil, Stephane Catharine; Soratto, Jacks; Gomes, Karin Martins; Amboni, Graziela; Souza, Caroline Zaneripe de. (2019). Use of Trilingual Posters as Access Technology to Prenatal of Immigrants. Am. In. Mult. J., October. (7) 4, 13-22.

Received: 07/29/2019

Accepted: 08/06/2019 